\title{
Design Strategies of Elderly Facilities Based on Existing Hotel Buildings Renovation
}

\author{
Chen Yang ${ }^{1}$, Sun Peiyang ${ }^{1, *}$, Li Xiangyun ${ }^{2}$ \\ ${ }^{1}$ School of Architecture, Harbin Institute of Technology, Harbin, China \\ ${ }^{2}$ Beijing Victory Star Architectural Design Co. Ltd, Beijing, China
}

Email address:

Chenyang1109@126.com (Chen Yang),18s134145@stu.hit.edu.cn(Sun Peiyang),31018351@qq.com (Li Xiangyun)

${ }^{*}$ Corresponding author

\section{To cite this article:}

Chen Yang, Sun Peiyang, Li Xiangyun. Design Strategies of Elderly Facilities Based on Existing Hotel Buildings Renovation——-Taking Beijing Friendship Health Community as an Example. Science Discovery. Vol. 7, No. 5, 2019, pp. 347-353. doi: 10.11648/j.sd.20190705.25

Received: November 4, 2019; Accepted: November 21, 2019; Published: December 3, 2019

\begin{abstract}
With the degree of population ageing rising continuously and the number of disabled and mentally disabled elderly rapidly increasing, the demand for care facilities for the elderly is growing. On the other hand, due to the Chinese urban expansion in the previous stage, a large number of existing buildings are faced with the problems of transformation and reconstruction, the reconstruction and utilization of existing building resources are also gradually paid attention to by all sectors of the society. In order to explore the new ideas of elderly facility development and realize the sustainable development of architecture. In this paper, through field research and case analysis methods, combined with the renovation process of Beijing friendship health community, put forward relevant strategies to transform the existing hotel buildings into care facilities for the elderly in terms of functional transformation, streamline arrangement, space construction, infiltration of the concept of suitable for the aged and improvement of structural adaptability, in the hope of raise rationalization proposals for the reconstruction of existing hotels into elderly facilities. The study also found that the existing hotel buildings have unique adaptability in the transformation into elderly facilities, which provides possible and convenient conditions for the success of the reconstruction.
\end{abstract}

Keywords: Elderly Facilities, Existing Hotel Buildings, Architecture Renovation, Suitable for the Aged

\section{基于既有酒店建筑改造的养老设施设计策略研究}

陈旸 $^{1}$, 孙培扬 ${ }^{*}$, 李祥云 ${ }^{2}$

${ }^{1}$ 哈尔滨工业大学建筑学院, 哈尔滨, 中国

${ }^{2}$ 北京维拓时代建筑设计股份有限公司, 北京, 中国

\section{邮箱}

chenyang1109@126.com (陈㽗),18s134145@stu.hit.edu.cn(孙培扬),31018351@qq.com(李祥云)

摘要：随着我国人口老龄化程度的不断加深和失能、失智老人数量的持续增多，对于养老设施的需求与日俱增。而 另一方面，由于我国前一阶段的城市扩张，大量既有建筑面临着转型和改造的问题，对于既有建筑资源的改造和利 用也逐步受到社会各界的重视。为了探求养老设施发展的新思路, 实现建筑的可持续发展。本文通过实地调研和案 例分析的方法, 结合北京友谊健康社区的改造过程, 提出了将既有酒店建筑改造为养老设施在功能转换、动线安排、 空间营造、适老化理念渗透以及提高结构适应性等方面的相关策略，以期对既有酒店改造为养老设施的建设提出合 理化建议。研究也发现既有酒店建筑在改造为养老设施方面具有独特的适应性, 为改造的成功进行提供了可能和便 利条件。 
关键词: 养老设施, 既有酒店建筑, 建筑改造, 适老化

\section{1. 引言}

当下, 我国巨大的养老压力和养老设施体系建设之间 的矛盾愈加突出。截至2018年底, 我国60岁及以上人口为 24949 万人，占总人口的 $17.9 \%[1]$, 其中失能、部分失能 老年人约有 4000 万人，预测到 2020 年，我国失能、部分失 能老年人将达到 4200 万人 $[2] 。$ 然而目前全国注册登记的养 老机构只有 2.9 万个, 各类养老机构提供的床位数总计约 714.2 万张 $[3]$, 相较于到 2020 年达到每千名老人拥有养老 床位35-45张的目标 [4], 缺口依然很大。既有酒店改造为 养老设施的优势主要在于可有效缓解我国严峻的养老问 题, 而且可以延长建筑的使用寿命, 对实现社会闲置资源 的价值重塑和建筑的可持续发展具有重要意义[5]。

北京友谊健康社区于2018年12月完成改造并进入试 运营。该项目是高级酒店改造为养老设施的典型案例, 其 设计和改造过程都体现了人性化和适老化的理念, 为养老 设施的发展提供了新的可能。

\section{2. 项目介绍}

\section{1. 基本概况}

友谊健康社区位于北京西城区西经路 11 号，地处北 京二环内的成熟居住区, 拥有着大量的潜在用户。目前
北京市 60 岁及以上户籍老年人口约 329.2 万人, 其中 16.49 万老年人已确定为失能老年人[6], 作为北京人口分布最 为密集的西城区, 户籍老年人口已达到 39 万多, 但是位 于二环内的养老机构只有 20家[7], 远不能满足北京核心 人口密集区的需求, 人口老龄化带来的这些问题显得更 为突出。

原有建筑新北纬饭店开业于1990年，是一栋地上 13 层、地下 2 层的四星级酒店,建筑面积 2.9 万平方米, 客房 384 间, 酒店内配有餐饮、会议中心和剧场等。设施改造后成 为活力长者公寓, 将原本的客房改造为589套养老居室, 其中自理区包含389套房间，照护区包含200套房。

\section{2. 选址及周边配套}

为了达到与医疗设施密切结合的目的, 并为老年人提 供良好的生活服务, 项目在选址时格外注重周边配套设施 的分布情况。立项之初首要考虑的因素是周边的医疗资源 配置情况, 以满足入住老年人的医疗需求。在基地东侧一 路之隔的就是北京友谊医院, 向北300米位置为宣武中医 院, 周边还有北京医院及宣武医院（如图1）。社区东临 西经路, 南侧有北纬路公交车站, 交通便利、可达性高, 周边餐饮、超市、银行、公园等基本生活配套设施也一应 俱全。

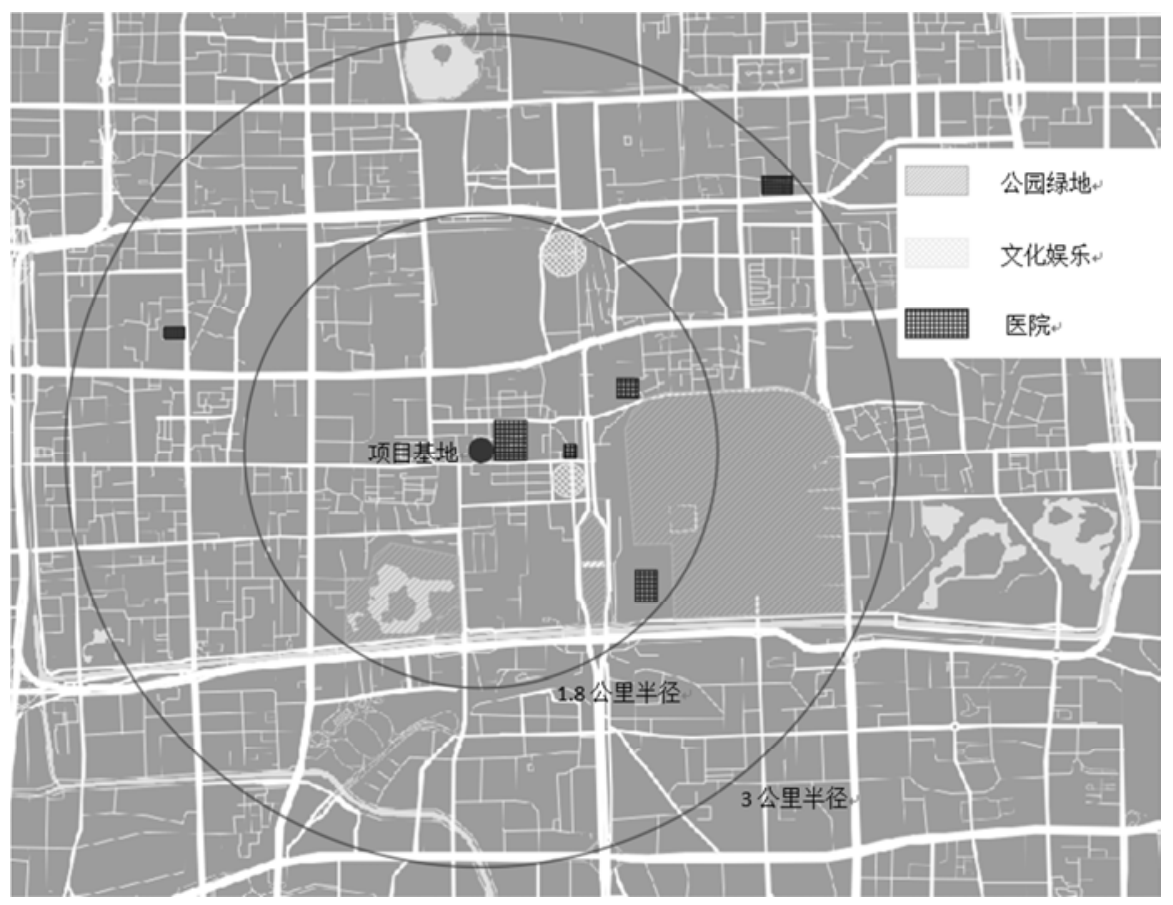

图1 北京友谊健康社区周边配套 图片来源: 作者自绘。

\section{3. 养老设施的功能转换策略}

将既有酒店建筑改造为养老设施的一个优势就是两 者在自身的功能属性和空间布局上有着极大的相似性, 都
主要是由私密的居住空间、开放的公共空间以及后勤管理 办公三个功能块形成主体功能框架。因此在改造中, 可以 依附原有的设计进行功能的转换和空间重构, 保持原有的 上层居住和底层公共属性不变, 从而减少大规模的拆改, 
做到快速集约的二次设计。但功能的转换过程中需要保证 各功能空间之间能够有效联系, 且避免不同功能属性空间 之间产生交叉和干扰[8]。

友谊健康社区的改造实践中, 核心交通核位置不变, 原4到13层的客房区通过简单改造成为全新的养老居室 (如图2), 但为了增强同层的邻里互动, 打破原本单调 的空间结构, 居住标准层内结合交通空间设置了邻里共享 空间以及洗衣房等小型生活辅助空间, 使原本单调的走廊 更富于变化和人情味儿。建筑底层保持了原有的公共和后 勤办公属性, 其中负二层依旧作为车库和后勤的功能使用;
负一层的会议、桑拿等功能空间被改造成室内生活街; 一 层保留了门厅和管理办公的主要功能, 但增添了老年人专 属的社交、会客以及健康服务空间; 二层原本的餐饮功能 被合理划分, 根据总需求量适当减少就餐面积, 结合采光 和朝向融入了文化娱乐的功能属性, 而且二层作为过渡层, 设置相对较安静的学习和餐饮空间, 既方便上下主要来往 人员的使用，也成为嘈杂空间与居住空间的分隔。

通过依托原有功能和空间重构, 不仅保证了整体功能 布局的合理性, 而且最大程度的减轻了改造强度, 节约了 时间和资金成本。

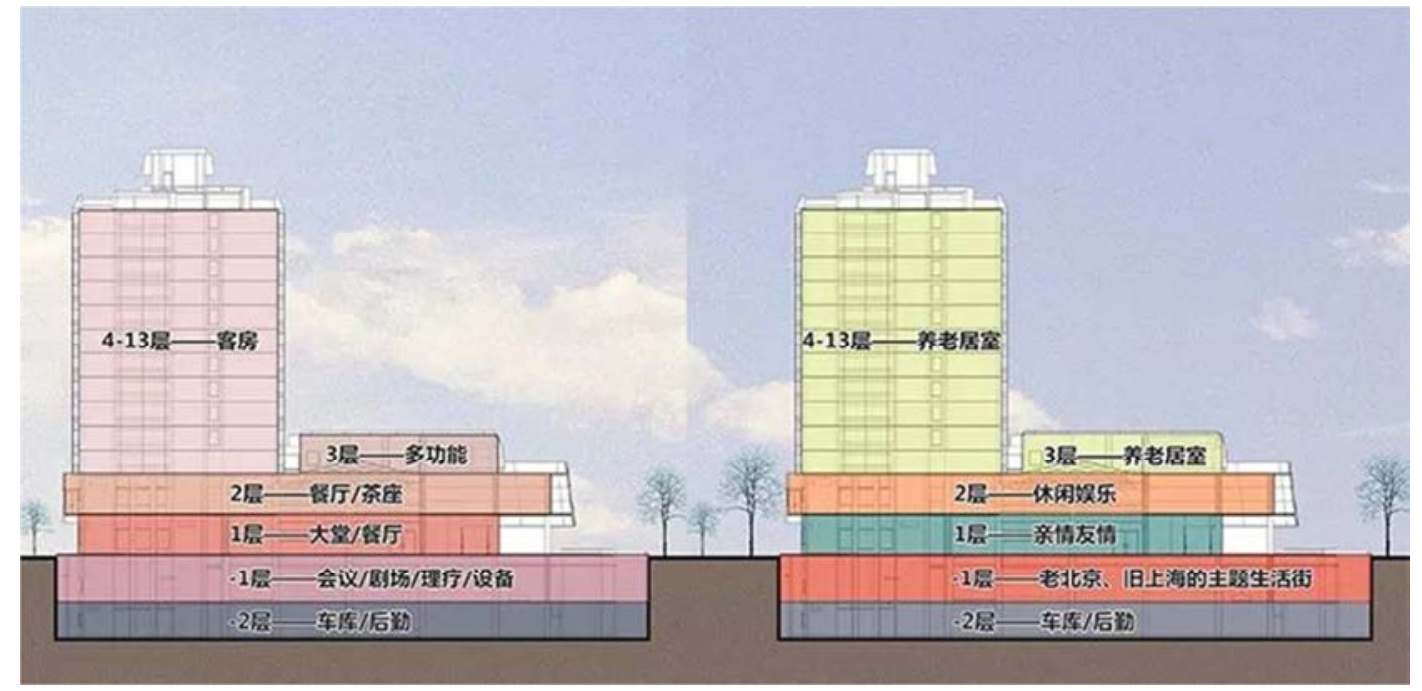

图2 功能转换对比图 图片来源: 首厚康健养老有限公司提供。

\section{4. 养老设施的多流线系统改造策略}

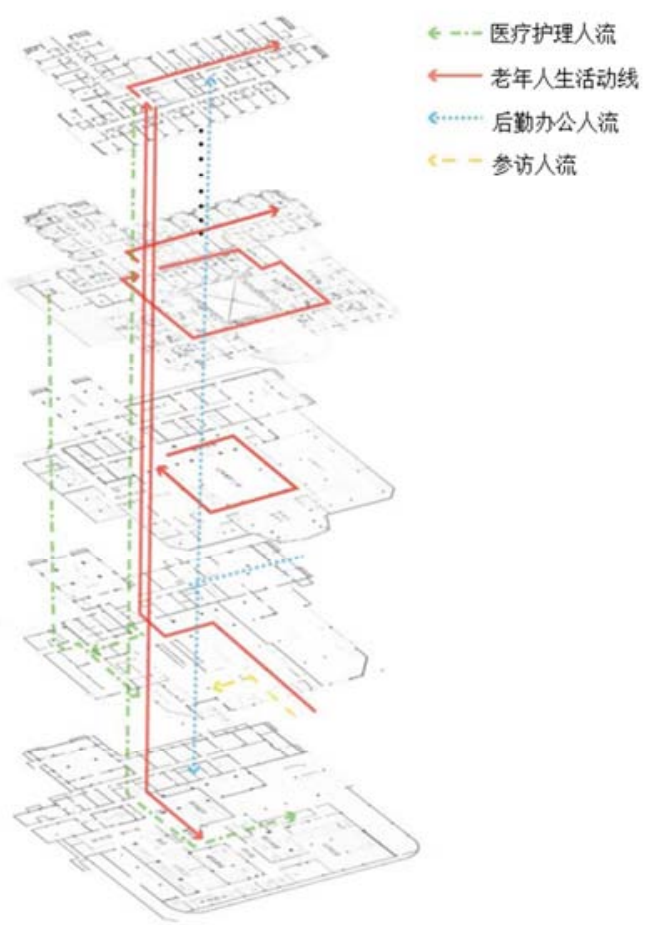

图3 改造后流线系统示意图 图片来源: 作者自绘。
作为主要针对特定人群服务的机构, 养老设施的流线 设计须以老年人的主要活动路径为主轴, 合理组织其他附 属服务动线。而酒店建筑本身也有着以住客为主的流线安 排, 因此在改造过程中可以尽可能保留原有主交通设置, 进行主体动线属性的替换, 并根据老年人的生理和心理特 点, 强化了老年人动线的可辨识性和便捷性, 并将附属服 务流线细分为: 参访人流、后勤办公人流以及医疗护理人 流, 利用原本的垂直交通设施, 围绕老年人生活动线形成 良好的服务动线体系（如图3）。

\section{1. 老年人生活动线的辨识性设计}

随着年龄的增长, 老年人的视力和记忆力呈现出不 断衰退状态, 因此对于入住老年人的生活动线设计应注 意提高其行动路线的辨识性, 保证老年人可以快捷到达 目的地。

改造设计从园区大门开始, 通过骑楼雨棚、门斗、入 口玄关、影壁等多重仪式感极强的空间元素将人流引导至 室内, 帮助老年人顺利找到归家之路。进入建筑内部之后, 改造设计将始于首层主入口骑楼雨棚至公寓主要客梯厅 的空间作为入住老人的主要动线, 入住老年人可由电梯直 达各自的居住楼层。在这条老年人归家的主动线沿途增加 了健康咨询区、健康体验区、便利店等日常便利服务功能。 不仅使老年人在出门和归家时能顺便得到及时的服务信 息, 而且增加了老年人与服务人员相互交流、相互了解的 机会。整条归家主动线通过强调不同层次的空间之间、不 
同私密程度的社交空间之间适宜的视线穿透，既为在此居 住的老年人创造不经意间偶遇的机会, 也保留了小聚的私 密氛围。最后通过电梯到达居住标准层之后，通过增设小 型共享空间，以及每间房门前的记忆盒，方便老年人找到 正确的居所。

\section{2. 后勤办公人流动线的独立性设计}

酒店和养老设施对于后勤管理和服务都有很高的要 求，既要及时的给予被服务对象优质的照顾，还要保证自 身拥有独立隐蔽的交通和运输系统。本项目沿用原酒店完 整的后勤办公流线, 将首层西侧的办公空间作为长者公寓 的后勤办公区。整个后勤办公区拥有自身独立的出入口和 后台空间，与一层大厅的公共空间互不干扰。后勤人员可 通过后勤电梯到达各层进行后勤服务, 避免和入住老年人 的生活流线产生不必要的交叉。

\section{3. 参访人流动线的便捷性设计}

对于外来的参访人员, 为避免其动线直接进入老年人 的生活圈, 同时为便于初来此地的参访人员快速到达接待 区, 本项目将主要的接待区设在一层入口大厅附近, 将原 有酒店前台接待及办公空间转换为参访接待空间, 同时在 接待区内设置了儿童活动区和访客接待洽谈区。将接待空 间设置于大厅一角, 增强其独立性, , 并便于健康社区的 集中管理和控制。

\section{4. 医疗护理人流动线的畅通性设计}

作为综合型养老设施, 让老年人得到及时的医疗救助 和日常健康护理是设计中的重点, 且需保障医疗护理流线 的快捷畅通[9]。但对于原酒店来说, 医疗救助的功能是缺 失的, 改造中需要重点增加医疗护理动线的设计。

本项目将原本一层南侧的商店改造为健康服务站, 使 老年人每天在门厅来往过程中得到及时的健康资讯, 当有 紧急情况发生时也可借由医疗电梯直达健康服务站, 再由 服务站直接对外的紧急出口将病人送往医院。地下一层原 本的会议空间被改造为康复和保健区, 由主电梯厅及一步 通向首层大厅的宽大楼梯将人流引入地下, 与地下生活街 相结合, 完善老年人的健康生活。

\section{5. 养老设施特色公共空间营造策略}

为满足老年人的个性化需要, 友谊健康社区结合动静 分区、使用频率及其采光和通风等条件, 对各层的公共空 间进行合理重塑，使二层的文化教育空间拥有良好的朝向 和视野; 一层为主串联各层的小型公共领域形成多层次的 共享空间; 充分利用既有资源将地下一层改造成为室内生 活街。

\section{1. 文化教育空间营造}

老年人对于学习新知识, 接触新伙伴, 形成新的学 习型团体具有较高的热情 [10]。改造设计中, 将采光和 视野良好、环境相对安静的二层公共区域用作主要的文 化教育空间。其中图书馆是由二层原有餐厅空间改造而 成。通过对项目整体居住人数、就餐率的估算, 适度缩 小原餐饮功能面积, 将各餐饮功能区向厨房方向收缩, 从而把东侧日照条件相对较好的空间改造为图书馆。图 书馆采用开放式书架和阅读区的布置形式, 烘托阅读和 交流氛围, 内部还设置了两间较为封闭的工作室兼小型 会议室, 为老人提供了继续工作和研讨的场所, 也可以 作为一些长者自发组织的小型学术社团活动的空间。二 层南侧集中改造成老年学堂, 学堂划分为若干可以适应 多种活动、学习情况的房间, 部分房间之间设置灵活隔 断, 以应对不同活动空间大小的需求。学堂内部形成独 立的动线系统, 避免外部人流的干扰, 保持安静的学习 活动氛围。

\section{2. 多层次、多尺度共享空间营造}

对于老年人来说, 交流与沟通是生活中排解情绪, 加 深感情的重要方式。因此在养老设施内设计多层次的共享 空间, 对于提升入住老年人的生活质量来说至关重要 [11]。 在友谊健康社区的设计中, 将底层的共享空间按照私密程 度的不同分为私密社交区、半私密社交区和开放社交区以 及封闭型的独立会客空间, 而高层的居住部分则每层都开 辟了邻里间的小型共享空间。

改造前的酒店首层除前台接待外, 主要的就是空旷 的大堂空间。大堂上部挑空, 由一个巨大的玻璃采光顶 覆盖, 整个大堂空间采光良好, 但大堂地面存在一定的 高差。为了更加方便老年人的活动, 改造中将原本大堂 地面的高差取消, 并建成具有文化氛围的公共开放空间, 不仅为老年人提供自由活动、交友的场所, 也为举行大 型集中活动预留了足够的空间(如图4)。一层中央空间形 成人流来往最频繁的开放社交区。一层开放社交区西北 侧结合水吧, 利用支撑柱和软隔断对空间进行半围合的 处理, 为一层大厅增加了半私密型的社交区, 为不同需 求的老年群体提供丰富的空间选择; 在一层大厅北部外 缘未通高的区域, 利用不同净高的限定形成了局部的私 密社交区。从开放到半私密, 从半私密到私密, 改造后 首层的公共区域为老年人创造了多种尺度的交往可能; 同时, 考虑到很多老年人退休后仍会有较多的社会活动 和私密访客接待的需求, 将首层西侧原本的酒吧改造为 一处私密的独立会客空间, 从而满足入住老年人延续社 会活动的行为需要。 


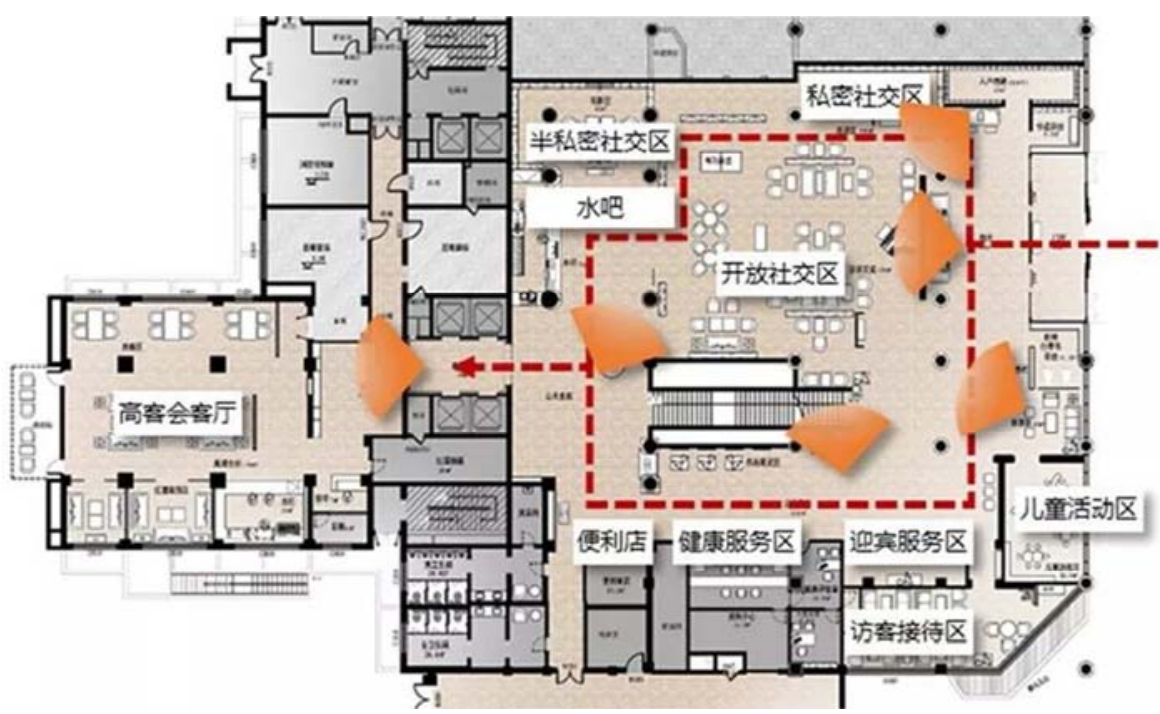

图4 一层交往空间示意 图片来源: 首厚康健养老有限公司提供。

\section{3. 室内生活街营造}

为了使老年人能够在养老设施内享受全面的生活配 套服务, 方便老年人及时解决生活中的基本需求, 在项目 内部设置相应的配套服务设施就显得尤为重要。原酒店建 筑地下一层主要功能包括商务会议区、天桥坊剧场、桑拿 SPA和辅助用房等, 内部环境比较昏暗, 走廊以尽端式为 主，很容易使老年人迷失方向。改造设计通过主电梯厅及 一步通向首层阳光厅的宽大楼梯将人流引入地下, 放宽与 垂直交通交接的区域, 有效避免了地下空间狭小阴暗的空 间感受。重新组织人流、明确动线方向后, 将走廊打通, 形成环形的室内步行街。
改造后仍保留了原有的区域文化色彩浓厚的天桥坊 剧场和一个多功能厅, 而将原会议区改造为文娱活动空间, 内部设有美容美发、运动健身、棋牌游乐、卡拉 $\mathrm{OK}$ 等设 施（如图5)。各功能为避免相互干扰, 采用通顶隔断墙 分隔, 但隔断材质选用半透明材质, 促进不同空间活动长 者的相互交流, 也可以降低服务人员的使用数量。原有的 桑拿SPA功能被老年人所需的健康咨询和中医理疗功能 所代替。利用原有空间和设备条件, 结合附近的运动健身 和康复厅形成了较为完整的健康管理功能板块, 从而强化 了机构整体的医疗和康复属性。

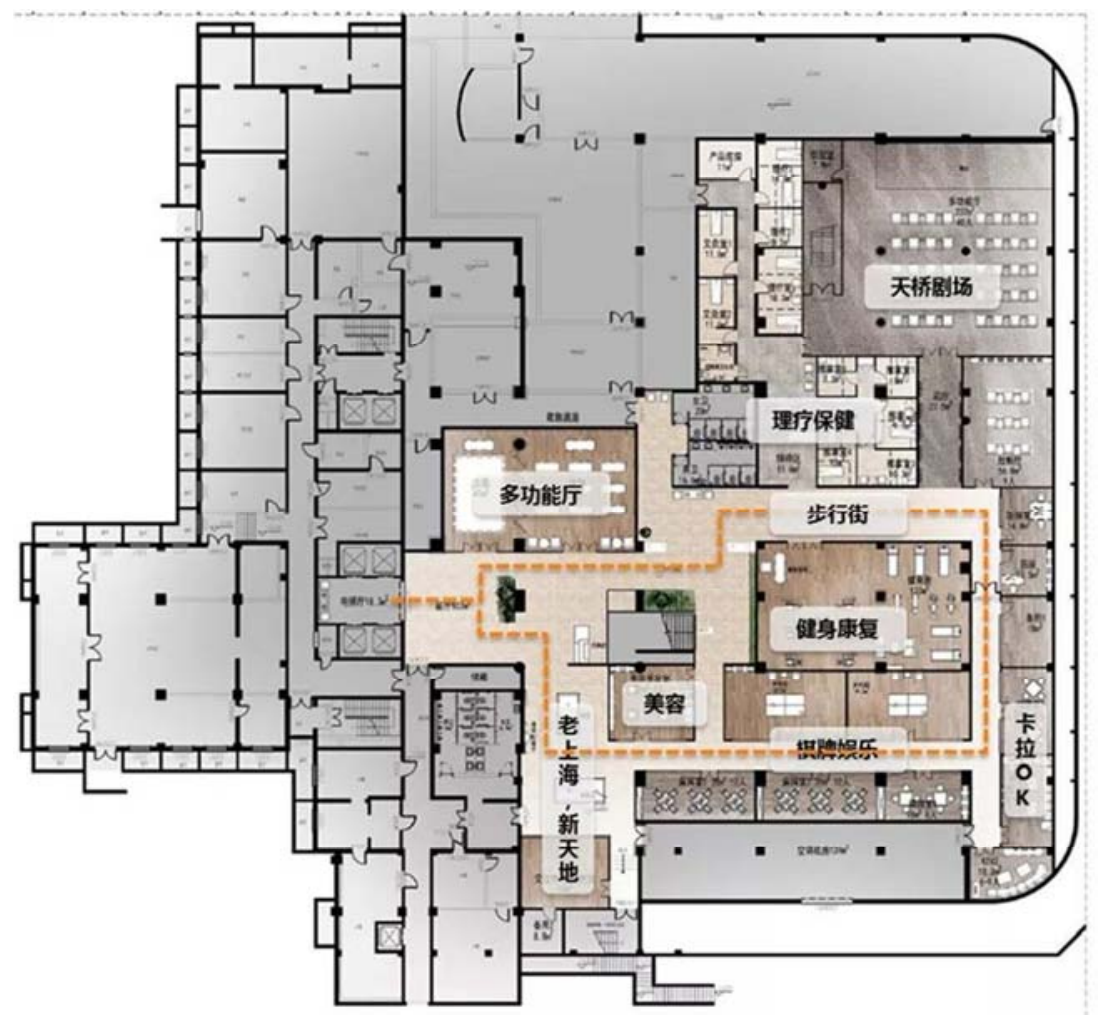

图5 负一层生活街示意 图片来源: 首厚康健养老有限公司提供。 


\section{6. 养老设施的结构适应性改造策略}

在友谊健康社区改造开始之前，首先对既有建筑结 构进行全面的预评估, 收集有关建筑结构现状、损坏和 老化程度等信息 [12]。从而建立全面的数据库和软件模型, 充分发挥不同结构的特点进行二次设计, 对薄弱环节进 行加固或改造, 提高结构的适应性和可持续性[13]。整体 未做大规模改造, 使建筑原有的结构骨架得以充分保留 和整修。

底层裙房部分充分利用框架结构空间给设计创造的 灵活性, 重新划分居住套型, 增加了面积段较大的套型产 品, 以弥补标准层因剪力墙结构限制全部套型均较小的问 题和产品面积段缺失的问题。

在标准层的整体改造中, 所有承重墙体得以最大限度 地保留。居室房间根据其所在的不同区域, 进行了重新梳 理, 局部利用原有结构条件, 进行重新划分。新的设计充 分利用原有房间面宽, 将房间内部功能分区进一步细化和 整合, 增加玄关空间、简厨操作台、外置手盆、衣帽柜、 飘窗休憩区等。将只能适应短期居住的空间改造提升为适 应老年人长期居住的空间。

对于改建建筑, 充分利用原有的结构, 可极大的 节约建设成本。提高结构的适应性, 不仅符合绿色低 碳, 节能减排的环保目标, 还能全面延长建筑的使用 寿命。

\section{7. 养老设施的适老化改造策略}

\section{1. 无障碍改造设计}

友谊健康社区的改造是以全方位的无障碍空间设计 为基础, 严格遵从规范设计要求, 把适老化的设计理念渗 透到老年人可能触碰到的每一个角落 [14]。

从方便老年人使用的角度出发, 整个社区室外道路进行 了平整化处理, 入口台阶处设计了严格符合规范要求的坡道。 对于室内的行进路线, 一层入口大堂原本的地面抬高被改造 成统一标高的平坦地面, 走廊全部采用无高差的设计, 同时 在两侧适宜高度处设置了连续的安全扶手。对于套内的无障 碍设计, 在老年居室中采用的是干湿分区的无障碍卫生间, 卫生间的推拉门也进行了无高差的处理, 最大限度的防止老 年人在使用洁具的过程中出现滑倒等危险, 洗面池也选用了 下部留空的处理, 方便坐轮椅老年人的使用 [15]。

\section{2. 精细化适老设计}

在友谊健康社区的改造设计中每一个细节都充分考虑 到老年人生活的舒适与便捷。机构内所有的家具都采用了全 环保材质的圆角设计家具, 以消除安全隐患。每位老年人居 室都在床头柜、淋浴区、马桶等位置设置了安全报警器（如 图6），保证老年人在发生意外时得到及时的救助。在居室 的入口处设计记忆盒, 可以摆放老人个性化的小物件, 方便 老人找到自己的居所。入户门采用单向的智能一卡通门锁, 保证私密与安全性的同时, 保证紧急逃生时的安全。

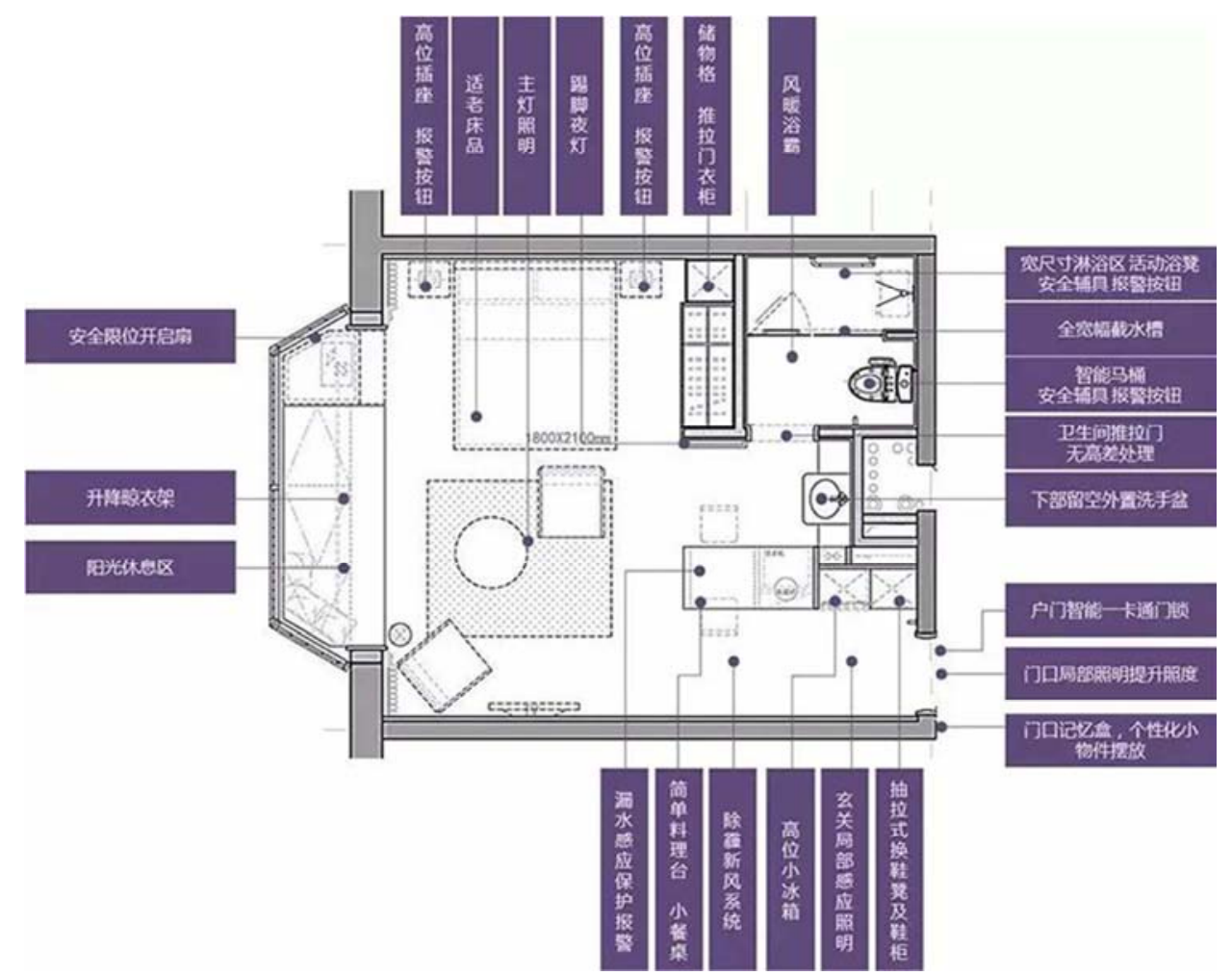

图6 居室精细化设计示意 图片来源: 首厚康健养老有限公司提供。 


\section{3. 智能化适老设计}

智能化的管理和控制系统不仅可以为老年人提供更 加准确及时的服务, 而且可以极大程度的减少人力成本, 提高整个系统的运行效率, 利于长期的运营[16]。

本项目在整个改造过程中引入了十余项智能化系统。 为了保证老年人的身体健康, 防止城市污染的入侵, 建筑 使用了新风除靁、中央空调系统; 为了保障老年人的人身 财产安全, 设置了入侵报警、视频监控等智能化系统，同 时借助移动互联网技术与安全照护工作的有机互联, 为老 年人的健康与安全保驾护航; 为了在保障正常使用的前提 下最大限度节约能源, 项目引进了国内先进的智慧照明系 统; 为了避免老年人滑倒, 在居室中央的料理台和小餐桌 下部设计安装了漏水感应保护报警装置, 在有漏水或地面 积水的情况发生时, 会及时发出警报声; 为了让老年人生 活的更加有品质, 改造中还融入了楼宇自控、无线网络、 可穿戴设备等智能化系统, 使得每一个细节都充分考虑到 老年人生活的舒适与便捷。

\section{8. 结论}

既有酒店建筑在改造成养老设施方面有着独特的适 应性。本文通过对北京友谊健康社区改造项目的深入分析, 从多个方面提出了将既有的酒店建筑改造为养老设施的 关键策略, 期望能够为未来的相关改造工程提供相关的借 鉴和建议。

(1) 在改造的过程中, 要始终将主要服务对象的需 求放在首位, 结合原设计进行整体功能的转换和主从流线 的安排。尤其要注重原本缺失的, 如医疗、会客等必要功 能属性和动线的重构, 做到功能布局合理、动静分区明确, 并保证老年人生活动线有清晰的辨识性、后勤办公人流动 线有完全的独立性、参访人流动线有高度的便捷性、参访 人流动线具有足够的通畅性。（2）为丰富设施内老年人 的生活, 可结合采光、通风、动静分区等实际条件营造多 层次共享空间、文化教育空间、室内生活街等特色公共 空间，以满足如今老年人更加多样化的生活需求。（3） 对于改造项目来说，通过预评估之后进行二次设计，并利 用原有结构灵活划分房间，不仅可以减轻改造成本，提高 结构适应性, 还有助于实现建筑的可持续发展。（4）要 将适老化的理念渗透到改造的全过程, 对建筑进行全方位 的规范化和便捷化的无障碍设计, 以及为进一步提高老年 人舒适性和安全性的精细化适老设计, 并利用智能化的手 段保障入住老年人的身心健康和生活服务系统的良好稳 定运行。

\section{致谢}

感谢首厚康健养老有限公司的全力支持与配合。

\section{参考文献}

[1] 李希如. 人口总量平稳增长城镇化水平稳步提高 [Z] http://www.stats.gov.cn/tjsj/sjjd/201901/t20190123_1646380. html。

[2] 龚维斌.社会体制蓝皮书: 中国社会体制改革报告NO.6 （2018）[M].北京：社会科学文献出版社,2018:124-439。

[3] 中华人民共和国国家发展与改革委员会.2017年养老消费 发展情况 (五)[Z]http://zhs.ndrc.gov.cn/gzyzcdt/201806/t20180629_891 344.html。

[4]国务院. 《关于加快发展养老服务业的若干意见》 [Z]]http://www.cctgroup.com.cn/zgjkyljtyxgs/zchb/zgzygwyg fxwj/zdyj/624517/index.html。

[5] 梁洋,毕既华. 推动我国既有建筑改造政策研究 [J].建设科 技,2010(22):78-80.

[6] 北京市老龄工作委员会办公室.北京市老龄事业和养老服 务 发 展 报 告 (2016 年 -2017 年 $)$ [Z]. http://www.bjmzj.gov.cn/templet/mzj/ShowArticle.jsp?id=126 949\&CLASS_ID=zhlm。

[7] 2017 年度中国养老机构发展研究报告( 内参) [Z]. https://www.docin.com/p-2142641579.html。

[8] 钱玉涛,刘峰,赵和生. 功能置换型既有建筑改造设计方法初 探——以南京市竹山小学原食堂改造为例 [J]. 江苏建 筑,2018(01):17-19.

[9] 林文洁,付北平.医养结合型养老机构医疗用房配置及空间 设计研究 [J].建筑学报,2018(S1):34-39.

[10] 王永梅.教育如何促进老年人使用社会养老服务?一一来自 北京的证据[J].兰州学刊,2018(11):187-198.

[11] 贾小叶. 既有社区公共建筑改造为养老建筑的设计探讨 [J]. 中国勘察设计,2018(08):89-90.

[12] Anne N. Nielsen Rasmus L. Jensen, Tine S. Larsen, Søren B. Nissen. Early stage decision support for sustainable building renovatione-A review [J]. Building and Environment, 2016, 103: $165-181$ 。

[13] 张立茂,吴贤国,张文静,陈虹宇,刘洋.BIM在既有建筑改造 设计中的应用研究[J].施工技术,2018,47(23):120-124.

[14] 徐伟琴. 居家养老模式下无障碍设计研究[D].无锡: 江南大 学,2018:92-93。

[15] 周燕珉. 老年住宅套内空间设计一卫生间篇[J].住区,2011, (05):106-115。

[16] 孙凌云,周雨薇.基于构建智能化养老服务系统的研究探讨 —以广东地区为例 $[J]$. 经贸实践,2018，(22):208-209. 